\title{
Effect Comparison of Rotated Tiles and Symmetrical Tiles on Binary Counter in DNA Self-Assembly
}

\author{
Aijun Zhu ${ }^{1,2,3}$, Chunxia Zhao ${ }^{1}$, Duanyong $\mathrm{Chen}^{1}$, Chuanpei $\mathrm{Xu}^{1}$, Cong $\mathrm{Hu}^{1}$ and Junhao $\mathrm{Niu}^{1,}{ }^{*}$ \\ ${ }^{1}$ School of Electronic Engineering and Automation, Guilin University of Electronic Technology, Guilin 541004, P.R.China \\ ${ }^{2}$ Department of Computer Science and Engineering, Texas A\&M University, College Station, TX, USA \\ ${ }^{3}$ Guangxi Key Laboratory of Automatic Detecting Technology and Instruments, Guilin 541004, P.R.China
}

\begin{abstract}
With the development of DNA Self-Assembly, several phenomena have happened and been discovered, which maybe lead to faulty crystals or patterns with faults. Among those unexpected phenomena, rotated tiles and symmetrical tiles have still not been discussed in detail. There is also no such research that compares the effect of rotated tiles and symmetrical tiles on Binary Counter in DNA SelfAssembly This paper compares and analyses the effect of rotated tiles and symmetrical tiles on binary counter in DNA Self-Assembly. The precise relationship between rotated tiles and symmetrical tiles is achieved taking into account the type of rotated tiles and symmetrical tiles. Experimental simulation results through Xgrow prove that the comparison and analysis is effective and correct.
\end{abstract}

\section{Introduction}

With the shrinking feature size of nano-fabrication, SelfAssembly is generally treated as an emerging subject and technology for its wide use and application in nanofabrication or computation [1,7-15]. In nanomanufacture system and algorithmic system, DNA is a basic element in DNA Self-Assembly. From a seed tile to final pattern, DNA is treated as a small and standard part and is used to set up a complex system or a unique pattern. Generally speaking, DNA Self-Assembly starts from a seed tile and goes on its growing by appending of several boundary tiles and rule tiles in one specific orientation. As we all know, it starts from the southeast corner to northwest. The attachment and appending of tiles is on the basis of a rigorous rule as to the growing corner and bonding feature. With the development of DNA SelfAssembly, several phenomena have happened and been discovered, which maybe lead to faulty crystals or patterns with faults [5]. Among those unexpected phenomena, rotated tiles and symmetrical tiles have still not been discussed in detail [3]. There is also no such research that compares the effect of rotated tiles and symmetrical tiles on Binary Counter in DNA SelfAssembly. In the case of rotated tiles and symmetrical tiles, several bonding action and behaviours are completely different from the original growth. In the case of rotated tiles and symmetrical tiles, the final patterns always differ from the reserved patterns; the reason is that the growth with rotated tiles and symmetrical tiles would conduct with uncertain ultimate patterns.

Generally speaking, the binary counter is a basic element and key unit in manufacture system of self- assembly and algorithmic system. There is a special tile set and its corresponding graphic pattern in the binary counter. For instance, literature [2] uses the binary counter for the component of a de-multiplexer. Literature [6] uses the binary counter for estimation framework. Literature [4] uses demultiplexers by self-assembly for memory design, while Literature [5] uses binary counter for assembly of a square. Literature [7] discusses the effect of rotated tile on binary counter. This paper mainly discusses the effect comparison of rotated tiles and symmetrical tiles on binary counter.

Organization of the rest of this paper is as follows. The mathematical model is briefly introduced in Section 2. The effect comparison of rotated tiles and symmetrical tiles on binary counter are presented in Section 3. The effect comparison of counting rules after single tile symmetry is introduced in Section 4. Finally, concluding remarks is given in Section 5.

\section{Mathematical model}

In order to establish the model of the growth behaviour, it is appropriate to apply a transition graph for ensuring the attachment in an established growth corner in DNA self-assembly. The transition graph could be defined as a directed graph $\mathrm{P}(\mathrm{D}, \mathrm{B})$. Let $\mathrm{D}$ be a vertex set and Let $\mathrm{B}$ be a directed edge set. Because the growth direction begins from south-east to north-west, without any doubt, the south-east corner should be defined as the growth corner. The directed edge set $\mathrm{B}$ is all the possible and feasible attachments in all growth corners. Therefore, every rule tile composes of two nodes and a directed edge. 
Definitely, there is special transition graph $\mathrm{P}$ in the binary counter tile set. The tile set of binary counter pattern is shown in Fig.1.
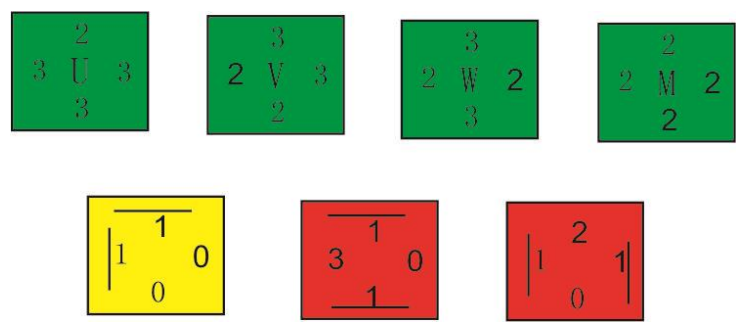

Fig. 1. All the tiles in the binary counter tile set.

There are three types of tile in binary counter which is rule tile, seed tile and boundary tile. However, growth is mostly obtained through rule tiles; there is the transition graph applied only for the rule tiles of binary counter in Fig.2. In binary counter, there is a seed tile $[(\mathrm{N}, \mathrm{E}, \mathrm{S}, \mathrm{W})][(1,0,0,1)]$ and two boundary tiles $[(2,1,0,1)$, $(1,0,1,3)]$. Rule tiles $[(2,3,3,3),(3,3,2,2), \quad(3,2,3,2)$, $(2,2,2,2)]$ are defined as $[\mathrm{U}, \mathrm{V}, \mathrm{W}, \mathrm{M}]$, respectively. In the case of counting, $\mathrm{V}$ and $\mathrm{W}$ are defined as digit " 1 ", and the corresponding $\mathrm{U}$ and $\mathrm{M}$ are defined as digit " 0 ". Under the assumption of fault-free attachment in the tile set, the counter number will increase one by each line from zero.

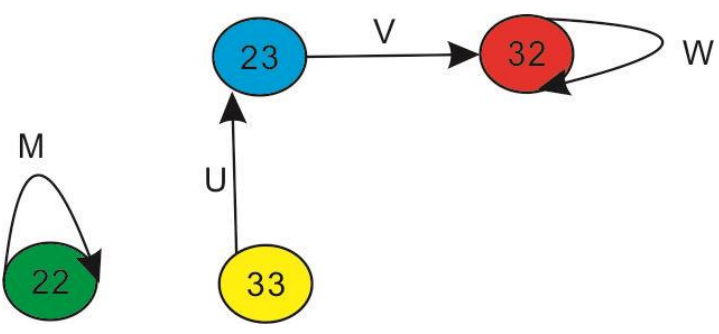

Fig. 2. Transition graph of binary counter tile set.

Let $\mathrm{P}^{\prime}\left(\mathrm{D}^{\prime}, \mathrm{B}^{\prime}\right)$ be symmetrical transition graph or rotated transition graph which is achieved by the symmetrical tiles or rotated tiles of the original rule tiles. Let $\mathrm{P}(\mathrm{D}, \mathrm{B})$ is the original transition graph. $\mathrm{X}$ symmetry of rule tile is the exchange of South with North. Y symmetry of rule tile is the exchange of East with West. XY symmetry of rule tile is both the exchange of North with South and the exchange of West with East. The rotation operations are defined as being in the clockwise direction and could be identified by the rotation angle(i.e., 90, 180, and 270 degrees). Therefore, the following situations should be taken into account as to the relationship between $\mathrm{P}$ and $\mathrm{P}$ '.

(1) $\mathrm{P}^{\prime}=\mathrm{P}$, which also means $\mathrm{D}^{\prime}=\mathrm{D}$ and $\mathrm{B}^{\prime}=\mathrm{B}$. In this case, symmetrical tile or rotated tile is exactly the same as the original tile. There are rule tiles $\mathrm{W}$ and $\mathrm{M}$ as to symmetrical tile, and there is rule tiles $M$ as to rotated tile.

(2) $\mathrm{P}^{\prime} !=\mathrm{P}$, to be exact, $\mathrm{D}^{\prime}=\mathrm{D}$ and $B^{\prime}=B \cup\{\forall b \mid b \notin B\}$. In such occasion, there are new edges without new vertexes which means no new growth corner. There are two subclasses. The first subclass is that the new edge connects two existing vertexes which are connected too in original $\mathrm{P}$. For instance, $\mathrm{X}$ symmetry of rule tile $U$ will lead to a new edge between nodes [2, 3] and [3, 3] in Fig.3 ; 90 degrees rotation of rule tile $U$ will lead to a new edge between nodes $[3,3]$ and $[3,2]$ in Fig.4. Therefore, transitions between some nodes possess several different choices. Generally, the final pattern would be not the same as what we wanted. The second subclass is that the new edge would connect two existing vertexes in $\mathrm{P}$ ' while no connection in P. For instance, $\mathrm{X}$ symmetry of rule tile $\mathrm{V}$ will lead to a new edge between nodes [3, 3] and [2, 2] in Fig.3; 90 degrees rotation of rule tile $U$ will lead to a new edge between nodes [3, 2] and [3, 3] in Fig.4. Therefore, transitions between such nodes are not sole. As a result, the final pattern would be not the same as what we wanted.

(3) $\mathrm{P}^{\prime} !=\mathrm{P}$, to be exact, $D^{\prime}=D \cup\{\forall d \mid d \notin D\}$ and $B^{\prime}=B$. The above occasion is out of question in theory. Generally speaking, the new node must posses an outedge. As a result, $B^{\prime}=B$ is out of question and null.

(4) $\mathrm{P}^{\prime} !=\mathrm{P}$, to be exact, $D^{\prime}=D \cup\{\forall d \mid d \notin D\}$ and $B^{\prime}=B \cup\{\forall b \mid b \notin B\}$. Such occasion most likely appears. There are also two subclasses. The first subclass is that the new nodes in the symmetrical transaction graph or rotated transaction graph perhaps be linked to the other nodes in P. Therefore, new edges are coming into being and the transitions between some nodes are not sole. As a result, the final pattern would be not the same as what we wanted. The second subclass is that the new nodes in the symmetrical transaction graph or rotated transaction graph are not connected to the other nodes in P. In such occasion, it is unlikely to get a new growth corner. Naturally, the final assembly must be what we want.

\section{Effects comparison on counting}

To compare the effects on counting, we should create symmetrical transition graph and rotated transition graph of binary counter tile set first. Symmetrical transition graph of the binary counter tile set is shown in Fig.3 and rotated transition graph of binary counter tile set of the binary counter tile set is shown in Fig. 4.

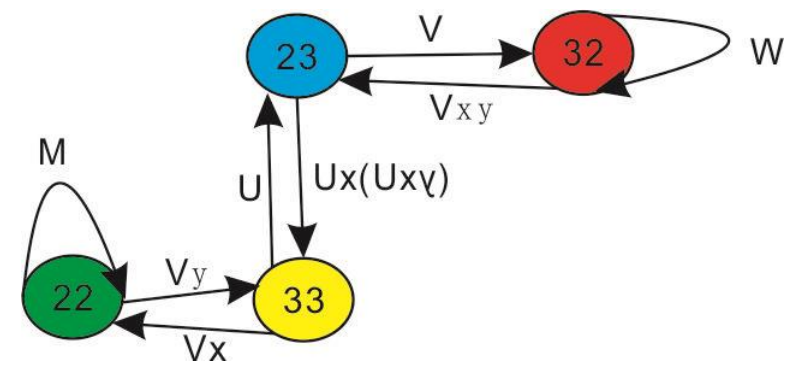

Fig. 3. Symmetrical transition graph of binary counter tile set. 


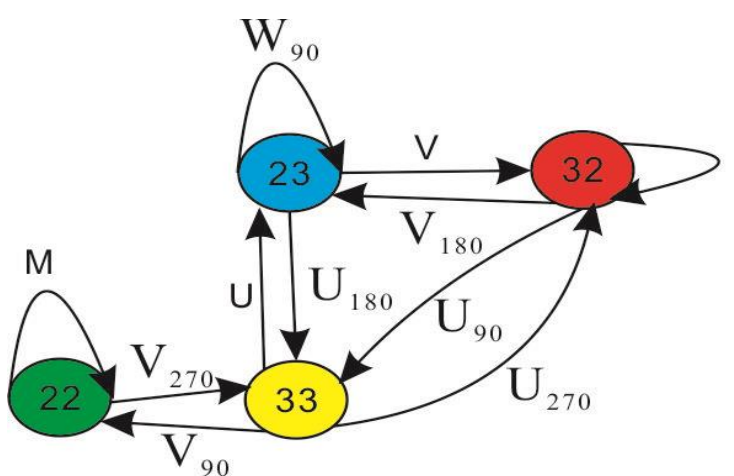

Fig. 4. Rotated transition graph of binary counter tile set.

As shown in Fig.3, Ux, Uxy, Vx, Vy and Vxy will result in new edges. While in Fig.4, V90, V180, V270, U90, U180, U 270 and W90 will result in new edges.

Compared the effects of rotated transition and symmetrical transition, we could conclude that rotation of tile $\mathrm{U}$ are the same as symmetry of tile $\mathrm{U}$ and rotation of tile $\mathrm{V}$ are the same as symmetry of tile V. As to tile $\mathrm{M}$, no matter what operation, it will not change at all. As to tile $\mathrm{W}$, it will not change at all for any symmetry. However, as to tile $\mathrm{W}$, it maybe change for some rotation. Generally speaking, the binary counter starts to increase by one each row from zero. Every row in the binary counter pattern denotes a count value. Let a tile be expressed by a four-tuple (N, E, S, W), which means the values of the bonds with east, south and west, respectively. Definitely, we can find exact relationship between each tile sequence and the counting value. Let the tile $\mathrm{V}(3,3,2,2)$ and the tile $\mathrm{W}(3,2,3,2)$ equal a binary value " 1 ", and let the tile $(2,2,2,2)$ and the tile $(2,3,3,3)$ equal a binary value " 0 ". As a result, symmetrical or rotated tile would cause a tile meaning " 1 " substituted by a tile meaning " 0 ". Of course, we can find other transaction which may be happen. For example, an X symmetry or 90 degrees rotation in tile $(3,3,2,2)$ (a digital " 1 ") would both produce a new tile $(2,3,3,2)$.

We would give a detailed example to demonstrate an exact occasion where a rotated tile or symmetrical tile could change the original final pattern.

\subsection{Both a rotated tile and symmetrical tile: leading to lower or smaller number}

In order to obtain the exact effect impact on counting, we can suppose current count value be 3 ("MMMMMMWV"or "00000011"). Naturally, the next fault-free count value should be 4("MMMMMVUU" or "00000100"). Fig.5 (a) demonstrate a segment of its selfassembly without any unwanted fault. Next step suppose a rotated tile and symmetrical tile occur. For example, a tile $\mathrm{V}(3,3,2,2)$ with $\mathrm{x}$ symmetry or 90 degrees rotation would produce a new tile $(2,3,3,2)$ with the same growth corner as the tile U. As a result, Vx or V90 is sure to attach in the place of the tile $\mathrm{U}$. This phenomenal will occur for the second $\mathrm{U}$ in the fourth row in the Fig.5 (a) in the fault free occasion, the tile sequence in the fourth row is "MMMMMVUU" or " 00000100 " in the Fig.5(a). With a rotated tile or symmetrical tile, the tile sequence is changed to be "MMMMMMVxU" or "MMMMMMV90U". Fig.5 (b) and Fig.5(c) show part of the final pattern under the condition of the rotated tile or symmetrical tile.

\begin{tabular}{|c|c|c|c|c|c|c|c|c|}
\hline Count Value: 60 & $\mathrm{M}$ & $\mathrm{M}$ & $\mathrm{M}$ & $\mathrm{M}=$ & $\mathrm{M}$ & We & $\mathrm{Ve}$ & UP \\
\hline Count Value: 54 & $\mathrm{M}$ & $\mathrm{M}$ & $\mathrm{M}$ & $\mathrm{M}+$ & $\mathrm{M}$ & We & $\mathrm{M}=$ & Vo \\
\hline Count Value: 40 & Mo & $\mathrm{M}$ & $\mathrm{M}$ & $\mathrm{M}=$ & $\mathrm{M}$ & $\mathrm{Ve}$ & UP & $\mathrm{U}$ \\
\hline Count Value: 3 & $\mathrm{M}$ & $\mathrm{M}$ & $\mathrm{M}$ & $\mathrm{M}+$ & $\mathrm{M}$ & $\mathrm{M}=$ & We & 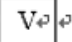 \\
\hline Count Value: 2 & $\mathrm{M}$ & $\mathrm{M}$ & $\mathrm{M}$ & $\mathrm{M} *$ & $\mathrm{M}$ & $\mathrm{M}+$ & $\mathrm{Ve}$ & 0 \\
\hline Count Value: & $\mathrm{M}$ & $\mathrm{M}$ & $\mathrm{M}$ & $\mathrm{M}$ & $\mathrm{M}$ & $\mathrm{M}$ & $\mathrm{M}$ & \\
\hline \multicolumn{9}{|c|}{ (a) } \\
\hline Count Value: 2 & $\mathrm{M}$ & $\mathrm{M}$ & $\mathrm{M}$ & $\mathrm{M}+$ & $\mathrm{M}$ & $\mathrm{M}$ & Ve & Us \\
\hline Count Value: 1 & $\mathrm{M}$ & $\mathrm{M}$ & $\mathrm{M}$ & $\mathrm{M}+$ & $\mathrm{Me}$ & $\mathrm{M}$ & $\mathrm{M}$ & 8 \\
\hline Count Value: & $\mathrm{M}$ & $\mathrm{M}$ & $\mathrm{M}$ & $\mathrm{M}+$ & $\mathrm{M}$ & $\mathrm{M}$ & $V x+$ & $\mathrm{UP}$ \\
\hline Count Value: & $\mathrm{Mp}$ & $\mathrm{M}$ & $\mathrm{M}$ & $\mathrm{M}=$ & $\mathrm{M}$ & $\mathrm{Mp}$ & We & $\mathrm{V}$ \\
\hline Count Value: & $\mathrm{M}$ & $\mathrm{M}$ & $\mathrm{M}$ & $M=$ & $\mathrm{M}$ & $\mathrm{M}^{p}$ & $\mathrm{~V}$ & $\mathrm{Uph}$ \\
\hline Count Value: & $\mathrm{M}$ & $\mathrm{M}$ & $\mathrm{M}$ & $\mathrm{M}+$ & $\mathrm{M}$ & $\mathrm{M}$ & $\mathrm{M}$ & \\
\hline \multicolumn{9}{|c|}{ (b) } \\
\hline Count Value: 2 & $\mathrm{M}$ & $\mathrm{M}$ & $\mathrm{M}$ & $\mathrm{M}+$ & $\mathrm{M}$ & $\mathrm{M}$ & Vo & Us \\
\hline Count Value: 1 & $\mathrm{M}$ & $\mathrm{M}$ & $\mathrm{M}$ & $\mathrm{M}+$ & $\mathrm{M} *$ & $\mathrm{Mp}$ & $\mathrm{M}$ & Ves \\
\hline Count Value : & $\mathrm{M}$ & $\mathrm{M}$ & $\mathrm{M}$ & $\mathrm{M}=$ & $\mathrm{M}$ & $\mathrm{Mp}$ & $V_{900}=$ & UP \\
\hline Count Value: & $\mathrm{M}$ & $\mathrm{M}$ & $\mathrm{M}$ & $\mathrm{M}=$ & $\mathrm{M}$ & $\mathrm{Mp}$ & We & $\mathrm{Ve}$ \\
\hline Count Value: 2 & $\mathrm{M}$ & $\mathrm{M}$ & $\mathrm{M}$ & $\mathrm{M}+$ & $\mathrm{M} *$ & $\mathrm{M}$ & Vo & U. \\
\hline Count Value: 1 & $\mathrm{M}$ & $\mathrm{M}$ & $\mathrm{M}$ & $\mathrm{M}=$ & $\mathrm{M}$ & $\mathrm{M}$ & $\mathrm{M}$ & $\mathrm{Ve}$ \\
\hline
\end{tabular}

Fig. 5. Part of Self-assembled pattern of binary counter (a) fault-free and (b) with a symmetric tile and (c) with a rotated tile

Compared Fig.5 (b) with Fig.5(c), we could find that a single Vx has the same effect with a single V90 on final pattern. They both lead to a lower number.

\section{Counting rules after one single symmetrical or rotated tile}

After the above consideration, we would give a discussion about counting rule in detail for binary counter with one single symmetrical and rotated tile. For simplification, suppose only o one single symmetrical or rotated tile on every row. Generally, every row in the binary counter pattern is divided into four parts. The value of the counter is shown in the following part. Because of one single symmetrical or rotated tile on every row, the counting value of is not $\mathrm{N}+1$. Suppose $\mathrm{j}$ is the exact position of symmetrical or rotated tile on every row. Such string "M..MW..WM..MW..WVU..U" in a row denotes a row in a binary counter. Let the right be the least significant digit and the left be the most significant digit. There is either zero number of " $U$ " or more, exactly one "V", either zero number of "W" or more, and either zero number of "M" or more. Above occasions contain all possible circumstances that maybe happen.

The difference between the counting value before (demonstrated as $\mathrm{N}$ ) and after one single symmetrical or rotated tile (demonstrated as Nnext) is shown in the 
following part. With each circumstance of single symmetrical or rotated tile, we will give a strict and detailed proof. There are four different circumstances.

1) Any V90 degrees rotation or Vx symmetrical tile: Nnext is in the range of 1 and $\mathrm{N}$.

Proof: first, let us take into account the case which V90 degrees rotation or $\mathrm{Vx}$ symmetrical tile would impact the "U" region that are seated in the least significant zeros and on the right side of the least significant ones. Such Nnext is at the right side. Let $\mathrm{j}$ equal 0 then $\mathrm{Nnext}=\mathrm{N}$.

Second, let the V90 degrees rotation or $\mathrm{Vx}$ symmetrical tile affect the most significant zero of the "U" region. Suppose the "U" regions are $t$ bits long, and we get

$$
\begin{aligned}
& \mathbf{N}=\mathbf{2}^{t}-1+\text { constant and } j=t-1 \\
& \mathbf{N}_{\text {next }}=\mathbf{2}^{t}-1+\text { constant }-\mathbf{2}^{t-1+1}+2=\text { constant }+1
\end{aligned}
$$

Where constant $=\sum b_{i} \times 2^{i} ; b_{i} \in\{0,1\}$ and $i>t$.

It is evident that $\mathbf{N}_{\text {next }}=$ constant $+1 \geq 1$.

2) Any $U 270$ degrees rotation or $U x$ (Uxy) symmetrical tile: Nnext is in the range of $\mathrm{N}+4$ and $3 * \mathrm{~N}+4$.

Proof: U270 degrees rotation and Ux or Uxy symmetrical tile would impact "V" region clearly as their growth corners are exactly the same as that of "V" region. First, as "V" region may be the least significant bit in the case of $\mathrm{j}$ equal zero, and $\mathbf{N}_{\text {next }}=N+\mathbf{2}^{0+1}+2=N+4$.

Second, let "V" region is the most significant bit 0 . Suppose the binary counter are $t$ bits long, and we get

$$
\begin{aligned}
& \mathbf{N}=\mathbf{2}^{t}-1+\text { constant and } j=t \\
& \mathbf{N}_{\text {next }}=N+\mathbf{2}^{t+1}+2 \leq 3 \times N+4
\end{aligned}
$$

It is evident $N+4 \leq \mathbf{N}_{\text {next }} \leq 3 \times N+4$.

3) Any V270 degrees rotation or Vxy symmetrical tile: Nnext is in the range of $\mathrm{N}+4$ and $2 *(\mathrm{~N}+1)$.

Proof: such V270 degrees rotation or Vxy symmetrical tile would impact "W" region clearly as its growth corner is the same as that of "W" region. First, as "W" region may be the least significant bit in the case of j equal one ("...WV"), and $\mathbf{N}_{\text {next }}=N+\mathbf{2}^{1}+2=N+4$.

Second, let "W" region be the most significant bit that is followed by "M", "V", and "U". Suppose the binary counter is t bits long, and we get

$$
\begin{gathered}
\mathbf{N}=\mathbf{2}^{t}-1+\text { constant and } j=t-1 \\
\mathbf{N}_{\text {next }}=N+\mathbf{2}^{t-1}+2 \leq 2 \times(N+1) \\
\text { It is evident } N+4 \leq \mathbf{N}_{\text {next }} \leq 2 \times(N+1)
\end{gathered}
$$

4) Any V90 degrees rotation or Vy symmetrical tile: Nnext is greater than or equal $\mathrm{N}+8$.
Proof: such V90 degrees rotation or Vy symmetrical tile would impact " $M$ " region clearly as its growth corner is the same as that of "M" region. First, as "M" region may be the least significant bit in the case of $j$ equal one (“...MU”), and $\mathbf{N}_{\text {next }}=N+\mathbf{2}^{1+1}+\mathbf{2}^{1}+2=N+8$

Second, as "W" region does not have the most significant bit of binary counter. Let the binary counter be $t$ bits long, and we get

$$
\begin{gathered}
\mathbf{N}=\mathbf{2}^{t}-1+\text { constant and } j=t \\
\mathbf{N}_{\text {next }}=N+\mathbf{2}^{t+1}+\mathbf{2}^{t}+2, \mathrm{t} \in[2,+\infty], \mathbf{N}_{\text {next }} \rightarrow+\infty
\end{gathered}
$$

It is evident $\mathbf{N}_{\text {next }} \geq N+8$.

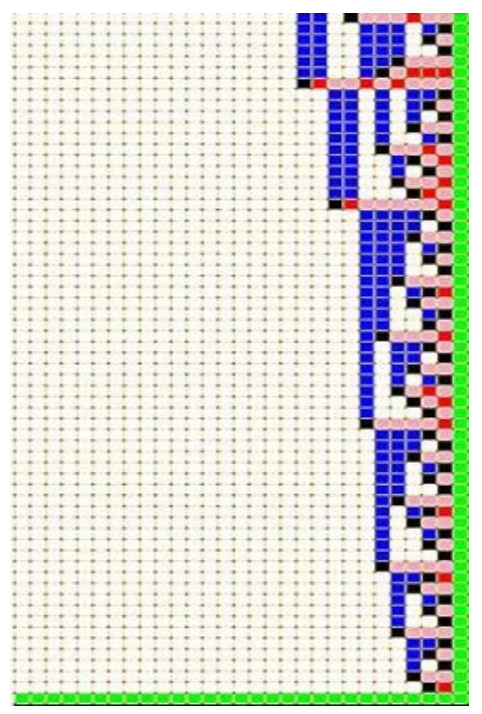

Fig. 6. $U_{90}$ degrees rotated tile or Ux (Uxy) symmetrical tile and its impact on binary counter.

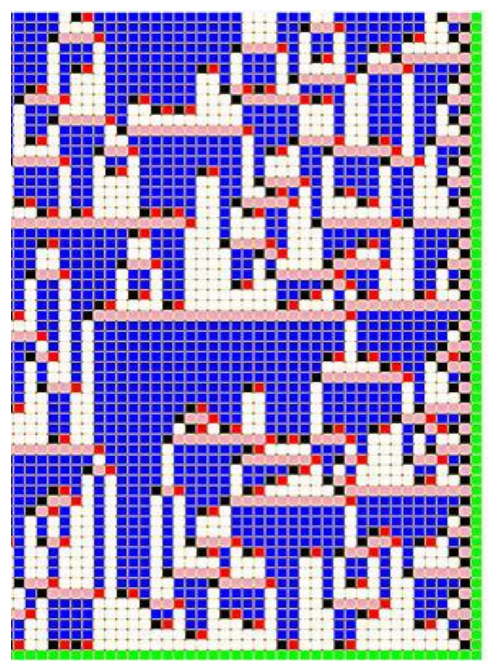

Fig. 7. $V_{180}$ degrees rotated tile or Vy symmetrical tile and its impact on binary counter.

Simulation results obtained by Xgrow prove that the related analysis is effective and errorless. Some sections of the simulation results are shown in Figs 6-7. 


\section{Conclusions}

We give detailed analyses for the effect comparison of rotated tile and symmetrical tile on binary counter. The detailed comparison of rotated tile and symmetrical tile on binary counter is obtained after taking into account the type of rotated tile and symmetrical tile. The analysis demonstrates that the final results are partly dissimilar with what we originally designed in the case of rotated tile and symmetrical tile because of the altered assembly mode. Simulation results from Xgrow show that the analysis is effective and correct.

\section{Acknowledgment}

This research has been partially funded by National Natural Science Foundation of China (61861012, 61561012, 61562013), Guangxi Natural Science Foundation Joint Funding Project (2018GXNSFAA138115), Guangxi Key Laboratory of Automatic Detecting Technology and Instruments (YQ16110), Talent Project of Guilin university of electronic technology (UF15008Y), Guangxi Natural Science Foundation (2017GXNSFAA198021, 2015GXNSFDA139030), China Scholarship Council (201508455020). Thanks Professor Fabrizio Lombardi, who is with Northeastern University, USA, for his valuable suggestions.

\section{References}

1. S. Zhang, D. M. Marini, W. Huang, and S. Santoro, "Design of nanostruc-Tured biological materials through self-assembly of peptides and proteins," Curr. Opin. Chem. Biol, vol. 6, 865-871(2002).

2. M. Cook, P. W. K. Rothemund, and E. Winfree, "Self-assembled circuitpatterns," DNA Computers 9, (Lecture Notes in Computing Science,vol. 2943). New York: Springer-Verlag, 91-107(2004).

3. F. Fujibayashi and S. Murata, "Precise simulation model for DNA tile self-assembly," IEEE Trans. Nanotechnol, vol. 8, no. 3, 361-368(2009).

4. M. Hashempour, Z. M. Arani, and F. Lombardi, "A graph model for tile sets in DNA self-assembly," in Proc. IEEE Int. Workshop Design Test Nano Devices, Circuits Syst, Boston, MA, 77-81(2008).

5. M.Hashempour,Z.M.Arani,andF.Lombardi,"Healing assessmentoftile sets for error tolerance in DNA self-assembly," IET Trans. Nanobiotech-nol, vol. 2, no. 4, 81-92(2008).

6. M. Y. Kao and R. Schweller, "Randomized selfassembly for approxi-mate shapes," in International Colloqium on Automata, Languages, and Programming (ICALP), NewYork:Springer-Verlag, 370-384(2008).

7. M. Hashempour, Z. M. Arani, and F. Lombardi, "Counting by DNA Self-Assembly in the Presence of Rotated Tiles," IEEE Trans. Nanotechnol, vol. 10, no. 3, 77-81 (2011).

8. J. Tan, X. Zhang and D. Liu, "Facile Preparation of CO2-Responsive Polymer Nano-Objects via Aqueous Photoinitiated Polymerization-Induced Self-Assembly (Photo-PISA)", Macromol Rapid Comm, vol. 38, no. 13, 15759-15765(2017).

9. K.T. Geun, P. Nokyoung, and K.U. Jeong, "Electrical Modulation of Graphene by the SelfAssembly of DNA-Functionalized Gold Nanoparticles," J Nanosci Nanotechno, vol. 17, no. 11, 8007-8011(2011).

10. E. Ducrot, M. He, G. Yi, "Colloidal alloys with preassembled clusters and spheres," Nat Mater, vol.16, no. 6, 652-657(2017).

11. S. Jiang, F. Hong, H. Hu, "Understanding the Elementary Steps in DNA Tile-Based SelfAssembly," ACS Nano, vol. 11, no. 9, 93709381(2017).

12. S. Dong, X. Ding, T. Guo, "Self-assembled hollow sphere shaped Bi2WO6/RGO composites for efficient sunlight-driven photocatalytic degradation of organic pollutants," Chem Eng J, vol. 316, no. 17, 778-789(2017).

13. D. Sun, T. Guo, B. Guan, "Counting by DNA Self-Assembly in the Presence of Rotated Tiles," J Lightwave Technol, vol.35, no. 16, 3354-3359(2017).

14. S. Xu, G. Ng, J. Xu, P. Rhiannon , Y. Jonathan and B.Cyrille,2-(Methylthio)ethyl Methacrylate: A Versatile Monomer for Stimuli Responsiveness and Polymerization-Induced Self-Assembly in the Presence of Air, ACS Macro Lett, vol. 6, no.11, 1237-1244(2017).

15. C. Zhu, S. Fu, J. Song, "Self-Assembled Fe-NDoped Carbon Nanotube Aerogels with SingleAtom Catalyst Feature as High-Efficiency Oxygen Reduction Electrocatalysts," Small, vol. 13, no. 15, 157-167(2017). 